\title{
Parents' intention to get vaccinated and to have their child vaccinated against COVID-19: cross-sectional analyses using data from the KUNO-Kids health study
}

\author{
Susanne Brandstetter ${ }^{1,2}$ (D) Merle M. Böhmer ${ }^{3,4} \cdot$ Maja Pawellek $^{1,2} \cdot$ Birgit Seelbach-Göbel $^{5} \cdot$ Michael Melter $^{1,2}$.

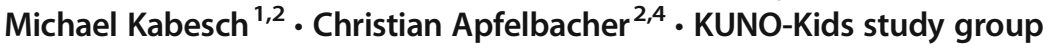

Received: 27 November 2020 / Revised: 1 April 2021 / Accepted: 29 April 2021 / Published online: 17 May 2021

(C) The Author(s) 2021

\begin{abstract}
A COVID-19 vaccine can be an important key for mitigating the spread of the pandemic, provided that it is accepted by a sufficient proportion of the population. This study investigated parents' intention to get vaccinated and to have one's child vaccinated against COVID-19. In May 2020, 612 parents participating with their child in the KUNO-Kids health study completed an online survey. Multivariable logistic regression models were calculated to analyze predictors of intention to vaccinate. Fifty-eight percent of parents intended to get vaccinated against COVID-19, and 51\% intended to have their child vaccinated. Significant predictors for the intention to get vaccinated and for having the child vaccinated included stronger parental confidence in one's knowledge about prevention measures and lower beliefs that policy measures were exaggerated.

Conclusion: COVID-19 vaccination hesitancy was considerable in our sample of parents in Germany. However, our study revealed some potentially modifiable factors which should be addressed by a comprehensive and tailored communication and education strategy.

\section{What is Known?}

- A COVID-19 vaccine can mitigate the spread of the pandemic.

- Many parents are skeptical about vaccinations in general.

What is New?

- COVID-19 vaccination hesitancy was considerable in our sample of parents from Germany, not only for getting vaccinated but also for having the child vaccinated.

- Negative parental attitudes regarding policy measures to contain the pandemic were associated with a lower intention to vaccinate.
\end{abstract}

Keywords COVID-19 · Vaccination · Vaccination hesitancy · Parents

Communicated by Nicole Ritz

Susanne Brandstetter

Susanne.brandstetter@barmherzige-regensburg.de

Merle M. Böhmer

merle.boehmer@1gl.bayern.de

Maja Pawellek

maja.pawellek@klinik.uni-regensburg.de

Birgit Seelbach-Göbel

birgit.seelbach-goebel@barmherzige-regensburg.de

Michael Melter

Michael.melter@ukr.de

Michael Kabesch

Michael.kabesch@barmherzige-regensburg.de
Christian Apfelbacher

Christian.apfelbacher@med.ovgu.de

1 University Children's Hospital Regensburg (KUNO), Regensburg, Germany

2 Member of the Research and Development Campus Regensburg (WECARE), Hospital St. Hedwig of the Order of St. John, Regensburg, Germany

3 Bavarian Health and Food Safety Authority, Oberschleissheim, Magdeburg, Germany

4 Institute of Social Medicine and Health Systems Research, Otto von Guericke University Magdeburg, Magdeburg, Germany

5 University Department of Gynecology and Obstetrics, Hospital St. Hedwig of the Order of St. John, University Medical Center Regensburg, Regensburg, Germany 


\section{Introduction}

The COVID-19 pandemic continues to threaten societies and their healthcare systems. Although safe and effective vaccines have been developed and approved for adults, major challenges remain, such as the provision of comprehensive access to vaccines and the reluctance of some people to get vaccinated.

In Western countries, a substantial proportion of the population is hesitant about vaccinations. For example, influenza vaccination uptake among pregnant women, healthcare workers, or the elderly is low in most European countries [7, 10]. Vaccination coverage is also insufficient in children. In Germany, despite many efforts at the end of the second year of life only $64 \%$ of children had received the second immunization against measles [11].

At the time of this study - considering this general vaccination hesitancy - it was questionable whether a sufficient proportion of people would intend to get vaccinated against COVID-19. On the one hand, the foreseeable fast development of COVID-19 vaccines was likely to elicit concerns. On the other hand, the pandemic attracted attention on an unprecedented scale, and people might be eager to protect themselves and others by getting vaccinated.

The aim of this study was to investigate the intention to get vaccinated and to have one's child vaccinated against COVID-19, thus contributing to the emerging body of evidence on people's vaccine acceptance. Using a large sample of parents who participate with their child in the KUNO-Kids health study prevalences and predictors of vaccination intention were analyzed.

\section{Methods}

\section{Design}

This study had a cross-sectional design. We used data collected in an online survey completed by parents participating in the KUNO-Kids health study between 5 and 28 May 2020 . The KUNO-Kids health study is a multipurpose birth cohort study situated in Eastern Bavaria (Regensburg), Germany. Women are approached during pregnancy or immediately after delivery and invited to participate in the study. Recruitment started in 2015 and is still ongoing. It is envisaged to followup children into young adulthood [4].

\section{Sample}

All participants in the KUNO-Kids study with a child between 1.5 and 5 years of age who had agreed to be contacted again were invited by mail to take part in the survey. One thousand and two hundred ninety-six families were contacted; 74 letters were not delivered because of invalid address.

\section{Measures}

\section{Outcomes}

This study investigated two outcomes: parents' intention to get vaccinated and to have their child vaccinated. ("If there was an effective vaccine against COVID-19, would you get vaccinated?,"... would you have your child vaccinated? -"yes", "no", "I don’t know"). Items were developed based on research on vaccinations against other infectious diseases. For the analyses, two categories were built (yes vs. no/I don't know).

\section{Predictor variables}

Variables considered as predictors included general characteristics (age of child and mother, parents' education (less than 10 years/10 years/more than 10 years of schooling), parents' country of birth (Germany/other)) and variables related to COVID-19 (informed by the COSMO survey [3]). Participants are asked whether family members/friends had a confirmed SARS-CoV-2 infection (yes, severe illness/yes, mild symptoms/no) or belonged to a risk group (yes/no), how much confidence they had in their knowledge about which safety measures were suitable for preventing infections (7 point Likert scale, not confident at all - very confident), whether they were concerned about their health or their family's health, whether they regularly sought information about the Corona crisis, how much they trusted in policy measures, and whether they considered policy measures to be exaggerated (all 5 point Likert scale, not at all - extremely) (see Table Supplement).

\section{Statistics}

A multivariable logistic regression model was performed for each of the outcome variables. Predictor variables were entered into multivariable models if they were associated with the outcome variable in a univariable logistic regression analysis (criterion: $p<.2$ ). All analyses were performed using SPSS.24. Odds ratios (ORs) with corresponding 95\% confidence intervals (CIs) were computed.

\section{Results}

Six hundred twelve families took part in the survey $(50.1 \%$ out of all families with valid addresses). Eighty percent of questionnaires were completed by mothers, $10 \%$ by fathers, and $10 \%$ by mothers and fathers together, respectively. 
Participants' children were at average 3.4 years old $(\mathrm{SD}=$ 0.9 ), in $11.9 \%$ of families at least one parent was born outside Germany, and in $78.1 \%$ of families at least one parent had attended school for more than 10 years. $24.4 \%$ reported that a family member or friend had been infected with SARSCoV-2.

Overall, $58 \%$ of parents intended to get vaccinated against COVID-19, and 51\% intended to have their child vaccinated. In the univariable models, for all predictor variables, the direction and size of the effect estimates were quite similar for the intention to get vaccinated and for the intention to have the child vaccinated, respectively (see Table 1).

In the multivariable models, a higher educational level (compared to a medium level) and stronger confidence in one's knowledge about prevention measures were associated with higher intention to get vaccinated and to have the child vaccinated, while stronger beliefs that policy measures were exaggerated were associated with a lower intention for both outcomes. Moreover, regular information seeking about the COVID-19 pandemic increased the intention to vaccinate the child, while the consideration of family or friends as risk group members decreased this intention (see Table 1).

\section{Discussion}

With only $58 \%$ of parents stating their intention to get vaccinated against COVID-19 and 51\% with the intention to have their child vaccinated, respectively, vaccination hesitancy was considerable in our sample of families in Germany. Also other studies showed that people were skeptical about a future COVID-19 vaccine. According to the representative

Table 1 Determinants of parents' intention to get vaccinated and to vaccinate the child: univariable and multivariable logistic regression analyses

\begin{tabular}{|c|c|c|c|c|c|c|c|c|c|c|c|c|}
\hline & \multicolumn{6}{|c|}{ Intention to get vaccinated } & \multicolumn{6}{|c|}{ Intention to vaccinate the child } \\
\hline & \multicolumn{3}{|c|}{ Univariable } & \multicolumn{3}{|c|}{ Multivariable } & \multicolumn{3}{|c|}{ Univariable } & \multicolumn{3}{|c|}{ Multivariable } \\
\hline & OR & $95 \% \mathrm{CI}$ & $p$ & OR & $95 \% \mathrm{CI}$ & $p$ & OR & $95 \% \mathrm{CI}$ & $p$ & OR & $95 \% \mathrm{CI}$ & $p$ \\
\hline Child's age (years) & 1.03 & $0.86-1.23$ & .767 & & & & 1.13 & $0.94-1.35$ & .191 & 1.16 & $0.91-1.36$ & .279 \\
\hline Mother' s age (years) & 1.06 & $1.02-1.11$ & .006 & 1.02 & $0.97-1.07$ & .509 & 1.04 & $1.00-1.09$ & .040 & 1.01 & $0.96-1.05$ & .919 \\
\hline $\begin{array}{l}\text { Low educational level } \\
\text { (<10 years of schooling) }\end{array}$ & 0.53 & $0.14-2.05$ & .358 & 0.98 & $0.23-4.23$ & .977 & 0.37 & $0.08-1.75$ & .209 & 0.59 & $0.11-3.06$ & .528 \\
\hline $\begin{array}{l}\text { Medium educational ( } 10 \\
\text { years of schooling) }\end{array}$ & Ref. & & & & & & Ref. & & & & & \\
\hline $\begin{array}{l}\text { High educational level } \\
\text { (university entrance level) }\end{array}$ & 2.86 & $1.88-4.32$ & $<.001$ & 2.70 & $1.70-4.28$ & $<.001$ & 2.37 & $1.56-3.60$ & $<.001$ & 1.99 & $1.26-3.34$ & .003 \\
\hline Migration background (yes) & 1.03 & $0.63-1.70$ & .90 & & & & 0.97 & $0.59-1.58$ & .892 & & & \\
\hline $\begin{array}{l}\text { No COVID-19 in family, } \\
\text { friends }\end{array}$ & Ref. & & & & & & Ref. & & & & & \\
\hline $\begin{array}{l}\text { COVID-19 with mild } \\
\text { symptoms in } \\
\text { family/friends }\end{array}$ & 1.42 & $0.89-2.25$ & .139 & 1.13 & $0.68-1.88$ & .631 & 1.30 & $0.83-2.04$ & .249 & 1.09 & $0.67-1.77$ & 0.74 \\
\hline $\begin{array}{l}\text { COVID-19 with severe } \\
\text { symptoms in } \\
\text { family/friends }\end{array}$ & 1.65 & $0.91-2.97$ & .098 & 1.42 & $0.73-2.76$ & .299 & 1.59 & $0.90-2.79$ & .110 & 1.35 & $0.72-2.54$ & .346 \\
\hline $\begin{array}{l}\text { Risk group member in family, } \\
\text { friends (yes) }\end{array}$ & 0.79 & $0.50-1.25$ & .317 & & & & 0.63 & $0.40-0.99$ & .047 & 0.59 & $0.36-0.99$ & .044 \\
\hline $\begin{array}{l}\text { Concerns about own health } \\
(0-4)\end{array}$ & 1.17 & $0.97-1.42$ & .097 & 1.12 & $0.87-1.44$ & .361 & 1.05 & $0.88-1.27$ & .575 & & & \\
\hline $\begin{array}{l}\text { Concerns about family health } \\
(0-4)\end{array}$ & 1.22 & $1.04-1.44$ & .016 & 1.08 & $0.87-1.34$ & .500 & 1.10 & $0.95-1.30$ & .202 & & & \\
\hline $\begin{array}{l}\text { Confidence in one's } \\
\text { knowledge about safety } \\
\text { measures }(0-6)\end{array}$ & 1.23 & $1.09-1.39$ & .001 & 1.21 & $1.05-1.39$ & .008 & 1.28 & $1.13-1.45$ & $<.001$ & 1.23 & $1.07-1.41$ & .003 \\
\hline Trust in policy measures $(0-4)$ & 1.69 & $1.4-2.02$ & $<.001$ & 1.19 & $0.94-1.51$ & .152 & 1.68 & $1.40-2.02$ & $<.001$ & 1.20 & $0.95-1.50$ & .127 \\
\hline $\begin{array}{l}\text { Perception that policy } \\
\text { measures are exaggerated } \\
(0-4)\end{array}$ & 0.50 & $0.41-0.60$ & $<.001$ & 0.58 & $0.46-0.73$ & $<.001$ & 0.54 & $0.45-0.65$ & $<.001$ & 0.60 & $0.49-0.76$ & $<.001$ \\
\hline $\begin{array}{l}\text { Regular information seeking } \\
\text { about Corona pandemic } \\
(0-4)\end{array}$ & 1.51 & $1.28-1.79$ & $<.000$ & 1.20 & $0.98-1.46$ & .073 & 1.52 & $1.28-1.80$ & $<.001$ & 1.22 & $1.00-1.48$ & .050 \\
\hline
\end{tabular}

Multivariable analysis: $N=600$; Nagelkerke's $R^{2}: .20 ; O R$ odds Ratio; $95 \% C I 95 \%$ confidence interval; $p$-value; ref. reference category; educational level of the higher educated parent; migration background if at least one parent was born not in Germany; bold: statistically significant $(p<.05)$ in the multivariable analyses 
COSMO survey in May 2020, about $60 \%$ of adults in Germany intended to get vaccinated [3]. This number continued to decrease until the end of 2020 and rose again with the second wave of COVID-19 infections and the start of the vaccination campaign in Germany to about $70 \%$ (March 2021) [2]. It will be crucial for the ongoing vaccination campaign to motivate also people who are considered at low risk for severe courses of COVID-19, e.g., young and healthy adults.

Until now, only a few studies addressed parents' intention to have their child vaccinated. In line with our results, a study from the UK with parents of children younger than 18 months found that they were more hesitant to have their child vaccinated than to get vaccinated themselves [1]. A multinational study [6] showed that parents were more willing to vaccinate their child when it was older. This suggests that particularly young children are considered to be more sensitive to possible side effects of vaccination. Other studies focused on the quality of the approval process for a COVID-19 vaccine for children: While Skjefte et al. found that a major reason for mothers' refusal of a COVID-19 vaccination for their child was concerns about insufficient data collection during the approval process [12], an international survey revealed that more than $40 \%$ of parents would accept even shortcuts in order to fasten the approval process for children [5]. In any case, once a COVID-19 vaccine will have been approved for children, special emphasis will be needed to convince parents that it is safe to have their child vaccinated.

When it comes to predictors of parents' intention to get vaccinated, our study yielded interesting results: the finding of a lower intention to have the child vaccinated if close ones are at risk for COVID-19 was unexpected. Maybe the attribution of a risk to others was accompanied by the perception that one's own family might be less affected. Parents' perception that political measures might be exaggerated was associated with lower intention for vaccination. Even before the COVID19 pandemic, people's trust in their governments was crucial: agreement with populist parties was associated with negative attitudes towards vaccination [8]. This finding - together with the significant effect of parents' education, regular information seeking and their confidence in knowledge about safety measures - emphasizes the importance of communication and education in order to address vaccination hesitancy.

Our study has some limitations: The survey was developed ad hoc and administered rapidly so that there was no space for validation and/or pretesting. It has also to be acknowledged that the situation regarding the spread of the COVID-19 pandemic, the associated policy measures, and the vaccination campaign is changing fast. We captured participants' views in May 2020, a period when it was still unclear whether and when vaccines would be available. Since then much research on the intention to get vaccinated against COVID-19 has been conducted. A rapid systematic review of 126 surveys from 31 countries found that many determinants of COVID-19 vaccination acceptance were universally relevant [9]. We also believe that associations between predictor variables and vaccination intention we found in our study might be a function of the prevalence of vaccination utilization in the population as being vaccinated will become the social norm.

\section{Conclusion}

In May 2020, the intention to get vaccinated oneself or have the child vaccinated against COVID-19 was low in our sample of parents in Germany. Our findings on predictors of intention to get vaccinated could contribute to the development of a comprehensive and tailored communication and education strategy.

Abbreviations CI, Confidence interval; COVID-19, Coronavirus disease 2019; OR, Odds ratio; Ref, Reference category; SARS-CoV-2, Severe acute respiratory syndrome coronavirus $2 ; \mathrm{SD}$, Standard deviation

Supplementary Information The online version contains supplementary material available at https://doi.org/10.1007/s00431-021-04094-z.

Acknowledgement We thank all families for their participation in the study and all members of the KUNO Kids study group: Andreas Ambrosch (Institute of Laboratory Medicine, Microbiology and Hygiene, Barmherzige Brüder Hospital, Regensburg, Germany), Petra Arndt (ZNL Transfercenter of Neuroscience and Learning, University of Ulm, Ulm, Germany), Andrea Baessler (Department of Internal Medicine II, Regensburg University Medical Center, Regensburg, Germany), Mark Berneburg (Department of Dermatology, University Medical Centre Regensburg, Regensburg, Germany), Stephan BöseO'Reilly (University Children's Hospital Regensburg (KUNO), Hospital St. Hedwig of the Order of St. John, Regensburg, Germany), Romuald Brunner (Clinic of Child and Adolescent Psychiatry, Psychosomatics and Psychotherapy, Bezirksklinikum Regensburg (medbo), Regensburg, Germany), Wolfgang Buchalla (Department of Conservative Dentistry and Periodontology, University Hospital Regensburg, University of Regensburg, Regensburg, Germany), Sara Fill Malfertheiner (Clinic of Obstetrics and Gynecology St. Hedwig, University of Regensburg, Regensburg, Germany), André Franke (Institute of Clinical Molecular Biology, Christian-Albrechts-University of Kiel, Kiel, Germany), Sebastian Häusler (Clinic of Obstetrics and Gynecology St. Hedwig, University of Regensburg, Regensburg, Germany), Iris Heid (Department of Genetic Epidemiology, University of Regensburg, Regensburg, Germany), Caroline Herr (Bavarian Health and Food Safety Authority (LGL), Munich, Germany), Wolfgang Högler (Department of Pediatrics and Adolescent Medicine, Johannes Kepler University Linz, Linz, Austria), Sebastian Kerzel (Department of Pediatric Pneumology and Allergy, University Children's Hospital Regensburg (KUNO), Hospital St. Hedwig of the Order of St. John, 
Regensburg, Germany), Michael Koller (Center for Clinical Studies, University Hospital Regensburg, Regensburg, Germany), Michael Leitzmann (Department of Epidemiology and Preventive Medicine, University of Regensburg, Regensburg, Germany), David Rothfuß (City of Regensburg, Coordinating Center for Early Interventions, Regensburg, Germany), Wolfgang Rösch (Department of Pediatric Urology, University Medical Center, Regensburg, Germany), Bianca Schaub (Pediatric Allergology, Department of Pediatrics, Dr. von Hauner Children's Hospital, University Hospital, LMU Munich, Munich, Germany), Bernhard H.F. Weber (Institute of Human Genetics, University of Regensburg, Regensburg, Germany), Stephan Weidinger (Department of Dermatology, Venereology and Allergy, University Hospital Schleswig-Holstein, Campus Kiel, Kiel, Germany), and Sven Wellmann (Department of Neonatology, University Children's Hospital Regensburg (KUNO), Hospital St. Hedwig of the Order of St. John, Regensburg, Germany).

Availability of data and material Data analyzed for this study can be obtained from the corresponding author on reasonable request.

Code availability Code for the analyses can be obtained from the corresponding author.

Authors' contributions Susanne Brandstetter designed the study, performed data analysis, interpreted the study findings, drafted the manuscript, critically evaluated the manuscript, and approved the final manuscript assubmitted. Merle Böhmer contributed to the design of the study, data analysis, manuscript writing, and revision of the manuscript. She critically evaluated the manuscript and approved the final manuscript as submitted. Maja Pawellek contributed to data collection, helped drafting the manuscript, critically evaluated the manuscript, and approved the final manuscript as submitted. Birgit Seelbach-Göbel contributed to data collection, critically evaluated the manuscript, and approved the final manuscript as submitted. Michael Melter contributed to data collection, critically evaluated the manuscript, and approved the final manuscript as submitted. Michael Kabesch designed the study, contributed to data collection, data interpretation, and drafting of the manuscript. He critically evaluated the manuscript and approved the final manuscript as submitted. Christian Apfelbacher designed the study, interpreted study findings, and drafted the manuscript. He critically evaluated the manuscript and approved the final manuscript as submitted.

Funding Open Access funding enabled and organized by Projekt DEAL. The KUNO-Kids study is funded by research grants of the EU (HEALS: 603946) and the German Federal Ministry for Education and Research (SYSINFLAME: 01ZX1306E). Further financial support was provided by the University Children's Hospital Regensburg (KUNO) and the hospital St. Hedwig of the order of St. John.

\section{Declarations}

Ethics approval The KUNO-Kids study was approved by the Ethics Committee of the University of Regensburg (Reference Number 14101-0347).

Consent to participate Written informed consent is required for all participants in the KUNO-Kids study. Participants can withdraw their consent at any time.

Consent for publication Not applicable
Conflict of interest The authors declare no competing interests.

Open Access This article is licensed under a Creative Commons Attribution 4.0 International License, which permits use, sharing, adaptation, distribution and reproduction in any medium or format, as long as you give appropriate credit to the original author(s) and the source, provide a link to the Creative Commons licence, and indicate if changes were made. The images or other third party material in this article are included in the article's Creative Commons licence, unless indicated otherwise in a credit line to the material. If material is not included in the article's Creative Commons licence and your intended use is not permitted by statutory regulation or exceeds the permitted use, you will need to obtain permission directly from the copyright holder. To view a copy of this licence, visit http://creativecommons.org/licenses/by/4.0/.

\section{References}

1. Bell S, Clarke R, Mounier-Jack S, Walker JL, Paterson P (2020) Parents' and guardians' views on the acceptability of a future COVID-19 vaccine: a multi-methods study in England. Vaccine. 38:7789-7798. https://doi.org/10.1016/j.vaccine.2020.10.027

2. Betsch C, Korn L, Felgendreff L, Eitze S, Schmid P, Sprengholz P, Wieler L, Schmich P, Stollorz V, Ramharter M, Bosnjak M, Omer S, Thaiss H, De BF, Von RU (2020) COVID-19 snapshot monitoring (COSMO Germany) - Wave 26. PsychArchives. https://doi.org/ 10.23668/PSYCHARCHIVES.4356

3. Betsch C, Wieler L, Bosnjak M, Ramharter M, Stollorz V, Omer S, Korn L, Sprengholz P, Felgendreff L, Eitze S, Schmid P (2020) Germany COVID-19 snapshot monitoring (COSMO Germany): monitoring knowledge, risk perceptions, preventive behaviours, and public trust in the current coronavirus outbreak in Germany. PsychArchives. https://doi.org/10.23668/psycharchives.2776

4. Brandstetter S, Toncheva AA, Niggel J, Wolff C, Gran S, SeelbachGöbel B, Apfelbacher C, Melter M, Kabesch M (2019) KUNOKids birth cohort study: rationale, design, and cohort description. Mol Cell Pediatr 6(1):1. https://doi.org/10.1186/s40348-018-0088$\mathrm{z}$

5. Goldman RD, Marneni SR, Seiler M, Brown JC, Klein EJ, Cotanda CP, Gelernter R, Yan TD, Hoeffe J, Davis AL, Griffiths MA, Hall JE, Gualco G, Mater A, Manzano S, Thompson GC, Ahmed S, Ali S, Shimizu N (2020) Caregivers' willingness to accept expedited vaccine research during the COVID-19 pandemic: a cross-sectional survey. Clin Ther 42(11):2124-2133. https://doi.org/10.1016/j. clinthera.2020.09.012

6. Goldman RD, Yan TD, Seiler M, Parra Cotanda C, Brown JC, Klein EJ, Hoeffe J, Gelernter R, Hall JE, Davis AL, Griffiths MA, Mater A, Manzano S, Gualco G, Shimizu N, Hurt TL, Ahmed S, Hansen M, Sheridan D, Ali S, Thompson GC, Gaucher N, Staubli G (2020) Caregiver willingness to vaccinate their children against COVID-19: Cross sectional survey. Vaccine 38(48): 7668-7673. https://doi.org/10.1016/j.vaccine.2020.09.084

7. Jorgensen $\mathrm{P}$, Mereckiene J, Cotter S, Johansen K, Tsolova S, Brown C (2018) How close are countries of the WHO European Region to achieving the goal of vaccinating $75 \%$ of key risk groups against influenza? Results from national surveys on seasonal influenza vaccination programmes, $2008 / 2009$ to $2014 / 2015$. Vaccine 36(4):442-452. https://doi.org/10.1016/j.vaccine.2017.12.019 
8. Kennedy J (2019) Populist politics and vaccine hesitancy in Western Europe: an analysis of national-level data. Eur J Pub Health 29(3):512-516. https://doi.org/10.1093/eurpub/ckz004

9. Lin C, Tu P, Beitsch LM (2020) Confidence and receptivity for COVID-19 vaccines: a rapid systematic review. Vaccines 9(1). https://doi.org/10.3390/vaccines9010016

10. Loerbroks A, Stock C, Bosch JA, Litaker DG, Apfelbacher CJ (2012) Influenza vaccination coverage among high-risk groups in 11 European countries. Eur J Pub Health 22(4):562-568. https:// doi.org/10.1093/eurpub/ckr094

11. Poethko-Müller C, Kuhnert R, Gillesberg Lassen S, Siedler A (2019) Durchimpfung von Kindern und Jugendlichen in Deutschland: Aktuelle Daten aus KiGGS Welle 2 und Trends aus der KiGGS-Studie (Vaccination coverage of children and adolescents in Germany: new data from KiGGS Wave 2 and trends from the KiGGS study). Bundesgesundheitsbl Gesundheitsforsch Gesundheitsschutz 62(4):410-421. https://doi.org/10.1007/ s00103-019-02901-5

12. Skjefte M, Ngirbabul M, Akeju O, Escudero D, Hernandez-Diaz S, Wyszynski DF, Wu JW (2021) COVID-19 vaccine acceptance among pregnant women and mothers of young children: results of a survey in 16 countries. Eur J Epidemiol 36:197-211. https://doi. org/10.1007/s10654-021-00728-6

Publisher's note Springer Nature remains neutral with regard to jurisdictional claims in published maps and institutional affiliations. 